\title{
Does the Conceivability of Zombies Entail Their Possibility?
}

\author{
Karol Polcyn*
}

Received: 11 July 2019 / Accepted: 10 February 2020

\begin{abstract}
According to the two-dimensional argument against materialism, developed by David Chalmers, the conceivability of zombies entails primary possibility, and the primary possibility of zombies entails further secondary possibility. I argue that the move from the conceivability to primary possibility of zombies is unjustified. Zombies are primarily impossible despite being conceivable if the corresponding phenomenal and microphysical concepts have coinciding primary intensions (refer to the same properties in all possible worlds considered as actual) despite being distinct concepts. But there is no good reason to think that phenomenal and microphysical concepts cannot have coinciding primary intensions despite being distinct concepts. As I argue, this conclusion follows from reflection on special cognitive features of phenomenal concepts.
\end{abstract}

Keywords: Conceivability; consciousness; phenomenal concepts; possibility; two-dimensional semantics.

\section{Introduction}

It seems that we can conceive of zombies: beings identical to us physically and functionally but lacking phenomenally conscious states. We are

* University of Szczecin

Institute of Philosophy and Cognitive Science, University of Szczecin, ul. Krakowska 71-79, 71-017 Szczecin, Poland

$\bowtie$ karol.polcyn@gmail.com

(C) The Author. Journal compilation (c) The Editorial Board, Organon F.

This article is distributed under the terms of the Creative Commons Attribution-NonCommercial 4.0 International Public License (CC BY-NC 4.0). 
phenomenally conscious in the sense that there is something it is like to undergo various experiences that we undergo, such as seeing red, feeling pain, being surprised, etc. By contrast, zombies have no such inner life. There is nothing it is like to be a zombie.

Assuming that zombies are conceivable, one crucial question is whether the conceivability of zombies entails that zombies are possible. Materialism is the view that zombies are impossible, which amounts to the view that consciousness is necessitated by physical properties. If the conceivability of zombies entails possibility, the conceivability of zombies entails that materialism is false. This issue has been central to the recent debate on the nature of consciousness.

The most influential argument to the effect that the conceivability of zombies entails possibility has been developed by David Chalmers. Chalmers $(1996,2010)$ articulates his argument in terms of two-dimensional semantics, by distinguishing two senses of the possibility of statements: $S$ is primarily possible if $S$ is true at a possible world considered as actual, and $S$ is secondarily possible if $S$ is true at a possible world considered as counterfactual. Chalmers argues that the conceivability of zombies entails that zombies are primarily possible. From this he infers further (under some qualifications to be clarified shortly) that zombies are secondarily possible.

Here I argue that the two-dimensional argument is far from successful. The argument seems to fail in its crucial step from the conceivability to primary possibility of zombies. In effect, the view that zombies are secondarily impossible despite being conceivable - in Chalmers' (2002a) classification, type-B materialism - remains unthreatened.

\section{The two-dimensional argument}

Assume that $P$ is a conjunction of all microphysical truths (including microphysical laws) and that $Q$ is the truth that someone is conscious. Then the claim that a zombie world is conceivable is the claim that $P \& \sim Q$ is conceivable. $P \& \sim Q$ says that everything is microphysically as in our world but that no one is conscious. In this sense, $P \& \sim Q$ says that the world is a zombie world. 
It is fair to assume that $P \& \sim Q$ is conceivable: $P \& \sim Q$ expresses a coherent hypothesis, that is, a hypothesis that cannot be ruled out a priori. The hypothesis is coherent, because there are no conceptual links between microphysical concepts, characterizing the world in terms of structure and dynamics, and phenomenal concepts, that is, the concepts of the phenomenal characters of experiences. ${ }^{1,2}$

Chalmers argues further that the conceivability of $P \& \sim Q$ entails that $P \& \sim Q$ is possible. At the most general level, the structure of his argument is as follows. The conceivability of $P \& \sim Q$ entails that $P \& \sim Q$ is primarily possible. But the primary possibility of $P \& \sim Q$ entails further - under certain assumptions about the nature of fundamental physical propertiesthat $P \& \sim Q$ is secondarily possible.

Chalmers' argument has shaped the debate on the nature of consciousness in the last two decades, and it continues to be puzzling to materialists. Is it right to think that the conceivability of $P \& \sim Q$ entails that $P \& \sim Q$ is primarily possible? Before evaluating the argument, let me present it in a more explicit way. We begin with a more explicit articulation of the key distinction between primary possibility and secondary possibility.

Intensions for statements are functions from possible worlds to truth values; correspondingly, intensions for concepts are functions from possible worlds to extensions. The primary intension of $S$ is true at a possible world $w$ ( $w$ verifies $S$, or $S$ is true at $w$ considered as actual) if and only if the following condition is met: if we came to accept that our world is qualitatively like $w$, we should endorse $S .{ }^{3}$ On the other hand, to say that the secondary intension of $S$ is true at $w$ is to say that $w$ satisfies $S$ in the sense that $S$ is true at $w$ considered as counterfactual. Correspondingly, $S$ is primarily possible (1-possible) if the primary intension of $S$ is true at some centered world, and $S$ is secondarily possible (2-possible) if the secondary

1 Following Chalmers (2002b), we can assume that $P \& \sim Q$ is ideally (as opposed to prima facie) conceivable: $P \& \sim Q$ cannot be ruled out a priori even on ideal rational reflection, that is, even when we abstract away from our cognitive limitations.

2 For a comprehensive defense of the conceivability of zombies against potential objections, see (Chalmers 2002a, 2010, sec. 4 and sec. 5).

3 Possible worlds considered as actual are centered worlds: worlds marked with a specified individual and time. 
intension of $S$ is true at some uncentered possible world. According to this framework, the negations of Kripkean a posteriori necessities are 2-impossible but not 1-impossible. For example, 'Water is not $\mathrm{H}_{2} \mathrm{O}$ ' is 2-impossible, but it is not 1-impossible. The primary intension of 'Water is not $\mathrm{H}_{2} \mathrm{O}$ ' is true at the centered possible world with XYZ in the oceans and lakes: if we came to accept that our world is the XYZ world, we should accept 'Water is not $\mathrm{H}_{2} \mathrm{O}$ ' (Chalmers 1996, ch. 2 and ch. 4, 2002b, 2006, 2010; Chalmers and Jackson 2001).

Given the above framework, Chalmers argues as follows. There is no gap between ideal conceivability and 1-possibility. In other words, ideal conceivability entails 1-possibility. If so, the ideal conceivability of $P \& \sim Q$ entails that $P \& \sim Q$ is 1-possible. If $P$ has the same primary and secondary intensions and $Q$ has the same primary and secondary intensions, it will follow that $P \& \sim Q$ is 2-possible. But here one must be careful. It is uncontroversial that $Q$ has the same primary and secondary intensions, because the concept of consciousness has the same primary and secondary intensions: the primary intension of the concept of consciousness picks out a phenomenal feel, and the secondary intension picks out a phenomenal feel as well. ${ }^{4}$ On the other hand, it is plausible to hold that the primary and secondary intensions of microphysical terms do not coincide - this will be the case if microphysical terms pick out the intrinsic properties that serve as the categorical bases of microphysical dispositions (Chalmers 2010, sec. 3). If one accepts this view, the primary and secondary intensions of $P$ will not coincide. And if the categorical bases of microphysical dispositions are phenomenal or protophenomenal properties, $P \& \sim Q$ will be 1 -possible but 2 -impossible. However, the view that the categorical bases of microphysical dispositions are phenomenal or protophenomenal properties (Russellian monism) can be left aside, given its highly speculative status. So, when this view is left aside, the 1-possibility of $P \& \sim Q$ entails 2-possibility. That is, when Russellian monism is left aside, the entailment from the 1-possibility to 2-possibility of $P \& \sim Q$ is justified even if the primary and secondary intensions of physical concepts do not coincide (even if $P$ has different primary and secondary

4 If something feels like a conscious experience, even in some counterfactual world, it is a conscious experience (Chalmers 1996, 133). By contrast, a substance that looks like water in a counterfactual world but is not $\mathrm{H}_{2} \mathrm{O}$ is not water.

Organon F 27 (3) 2020: 395-410 
intensions). In the light of the above analysis, the two-dimensional argument can be articulated as follows (Chalmers 2010):

1. $P \& \sim Q$ is conceivable.

2. If $P \& \sim Q$ is conceivable, $P \& \sim Q$ is 1-possible.

3. If $P \& \sim Q$ is 1 -possible, $P \& \sim Q$ is 2-possible.

4. If $P \& \sim Q$ is 2-possible, materialism is false.

5. So, materialism is false.

A note on the intensions of microphysical concepts. As Chalmers (2002a, sec. 11) points out, whether microphysical terms have different primary and secondary intensions, namely, whether those terms pick out intrinsic properties rather than microphysical dispositions is a terminological rather than a substantive issue. If so, we will stipulate here that the primary and secondary intensions of microphysical concepts coincide. This will simplify the subsequent discussion.

Now, how does Chalmers justify premise 2? Premise 2 follows from the general principle that if $S$ is ideally conceivable, $S$ is 1-possible. This principle (CP) comes down to the point that there is no gap between conceivability and 1-possibility. Chalmers (2010, sec. 8 ) points out that there are no clear counterexamples to CP. In particular, the principle is consistent with Kripkean a posteriori necessities, as we saw earlier. But why suppose that CP must be true? To justify this, Chalmers argues that the view that there is a gap between conceivability and 1-possibility amounts to the unacceptable view that a space of metaphysically possible worlds is narrower than the space of ideally conceivable worlds. Chalmers' reasoning goes here as follows. For any sentence $S$, if $S$ cannot be ruled out a priori, there is a scenario (a maximal, a priori coherent hypothesis about the character of the actual world) that verifies $S$ in the sense that it would be incoherent to suppose that the scenario obtains and $S$ is not true (Chalmers 2002b, 2006, 2010, sec. 7). For example, the scenario verifying 'Water is not $\mathrm{H}_{2} \mathrm{O}$ ' involves the assumption that the transparent liquid that fills the oceans and lakes is not $\mathrm{H}_{2} \mathrm{O}$ but, say, XYZ: if we suppose that this scenario actually obtains, we should accept 'Water is not $\mathrm{H}_{2} \mathrm{O}$.' If $S$ is conceivable and 1possible, there is a possible world that corresponds to the scenario that verifies $S$. The possible world at issue is the world that verifies $S$. But if $S$ is both conceivable and 1-impossible, there is no possible world corresponding 
to the scenario that verifies $S$ (Chalmers 2010, sec. 7). Chalmers (2010, sec. 10) argues then that there cannot be scenarios that correspond to no possible worlds. As he points out, we have no concept of independent metaphysical modality, introducing a separate space of metaphysically possible worlds, in addition to logically possible worlds. Independent metaphysical modality plays no role in our conceptual system.

\section{The unjustified move from the conceivability to primary possibility of zombies}

In reply to the two-dimensional argument, I take it that premise 2 is unjustified. Leaving the details of how Chalmers justifies this premise aside, it is arguable that there is no good reason to think that $P \& \sim Q$ cannot be 1-impossible despite being conceivable. We can see this if we articulate the content of the claim that $P \& \sim Q$ is 1-impossible despite being conceivable in terms of the intensions of phenomenal and microphysical concepts.

What are the conditions under which it is true that $P \& \sim Q$ is 1-impossible despite being conceivable? Well, $P \& \sim Q$ is 1 -impossible despite being conceivable if the corresponding phenomenal and microphysical concepts have coinciding primary intensions despite being distinct concepts, that is, if they refer to the same properties in all possible worlds considered as actual. This can be analyzed further. To say that two distinct concepts have coinciding primary intensions is to say that two distinct concepts (i) corefer and (ii) are epistemically rigid in the sense that each concept picks out the same thing in all possible worlds considered as actual. If so, we can say that $P \& \sim Q$ is 1-impossible despite being conceivable if the corresponding phenomenal and microphysical concepts corefer, are distinct and epistemically rigid.

Now, phenomenal and microphysical concepts are, by assumption, a priori distinct. They are also epistemically rigid. As Chalmers (2003) points out, each phenomenal concept picks out one and the same property in all possible worlds considered as actual. The same can be said about microphysical concepts. The idea that microphysical concepts are epistemically rigid corresponds to our earlier assumption that phenomenal concepts have coinciding primary and secondary intensions. As we saw, this assumption 
is not true if we accept Russellian monism. However, here we are leaving Russellian monism aside.

Given that phenomenal and microphysical concepts are a priori distinct and epistemically rigid, the key question is whether they can corefer despite being distinct epistemically rigid concepts. In other words, the question is whether those concepts can refer epistemically rigidly to the same thing despite being distinct concepts. If they can, this will imply that the primary conceivability of $P \& \sim Q$ does not entail 1-possibility.

I take it that there are no uncontroversial examples of conceptually distinct epistemically rigid concepts of the same property. Typically, a posteriori true identity claims involve at least one concept that is not epistemically rigid. This is obviously true in the case of Kripkean a posteriori identities, such as 'Water is $\mathrm{H}_{2} \mathrm{O}$ ' or 'Cicero is Tully.' Materialists responding to the conceivability argument have proposed various examples of identity claims that would break this rule. However, it seems that all such attempts are far from uncontroversial. For example, Kallestrup (2006) considers the identity claim ' $Q=\mathrm{H}_{2} \mathrm{O}$ ', where $Q$ is a quantum-mechanical description of $\mathrm{H}_{2} \mathrm{O}$, and argues that this claim is both true a posteriori and 1-necessary. Thus, Kallestrup assumes that $Q$ and $\mathrm{H}_{2} \mathrm{O}$ are distinct epistemically rigid concepts of the same thing. In reply, Chalmers $(2010,171-72)$ argues, however, that $Q$ is not epistemically rigid: it is plausible that something with the structure of $Q$ yields different chemical-level properties due to different quantum-mechanical laws. ${ }^{5}$

Still, the fact that there are no uncontroversial cases of conceptually distinct epistemically rigid concepts of the same thing does not give us the reason to think that there cannot be such concepts. If one thinks that there cannot be such concepts, one would need to show this on independent grounds, by providing some principal reason why conceptually distinct epistemically rigid concepts can never refer to the same thing. Is it plausible to think that there such a reason? In my view, there is no such reason. As I argue below, there is no good reason to think that phenomenal and microphysical concepts cannot be conceptually distinct epistemically rigid

5 For Chalmers' replies to other proposed examples of strong necessities (statements that are a posteriori and 1-necessary), see (Chalmers 2010, sec. 8). 
concepts of the same thing. This conclusion follows from reflection on special cognitive features of phenomenal concepts.

To see one potential reason why phenomenal and microphysical concepts cannot refer to the same thing, compare epistemically rigid concepts with concepts that are rigid without being epistemically rigid, such as the concept water. The concept water refers to different kinds in different possible worlds considered as actual, which means that we cannot know a priori what it refers to in the actual world. What this concept refers to depends on how the world turns out. Things are different with epistemically rigid concepts, that is, concepts referring to the same property in all possible worlds considered as actual. The reference of such concepts does not depend on how the world turns. Thus, in the case of epistemically rigid concepts, we know a priori what they refer to.

Given that epistemic rigidity has the above implication, it is natural to expect that epistemically rigid concepts referring to the same property will not be a priori distinct. In particular, it might seem that we should expect that if phenomenal and microphysical concepts refer to the same thing, there should be an a priori connection between those concepts. But, of course, it is not true a priori that phenomenal and microphysical concepts corefer. In effect, given our expectation, it would follow that those concepts cannot corefer.

I reply, I do not think that this argument succeeds. Call the expectation that epistemically rigid concepts referring to the same property cannot be a priori distinct 'the expectation of transparency.' I take it that the expectation of transparency is unjustified in the case of the corresponding phenomenal and microphysical concepts. This has to do with the fact that phenomenal and microphysical concepts play very different cognitive roles. Microphysical concepts analyze their referents in theoretical (scientific) terms. But this is not how phenomenal concepts work. Phenomenal concepts do not analyze their referents in theoretical terms. Instead, we use them to refer to our own conscious experiences when we actually undergo those experiences or when we recreate them in imagination. ${ }^{6}$ If this is so,

${ }_{6}$ Some philosophers (e.g. Chalmers 2007; Levine 2007) assume that phenomenal concepts acquaint us with conscious experiences in the sense that we gain substantive knowledge about our own experiences merely in virtue of undergoing (or imagining)

Organon F 27 (3) 2020: 395-410 
then we cannot expect to be able to see a priori that phenomenal and microphysical concepts corefer, even if they do as a matter of facts. The different cognitive roles of those concepts will keep them unconnected a priori, even if both concepts refer - epistemically rigidly - to the same thing. ${ }^{7}$

Interestingly, Papineau (2007, 128-32) has another way of explaining why the expectation of transparency in the case of phenomenal and microphysical concepts is unjustified. He argues that it simply does not follow, from the fact that phenomenal concepts are epistemically rigid, that we should be able to see a priori that phenomenal concepts refer to microphysical properties if they do. We can explain why phenomenal concepts are

them. However, most of physicalists would not go that far. Instead, they assume simply that phenomenal concepts are exercised in the presence of the experiences being referred to or their imaginative recreations (see Loar 1997; Papineau 2002, 2007). Loar assumes that this is consistent with the idea that phenomenal concepts are type-demonstratives. On the other hand, Papineau assumes that, insofar as phenomenal concepts are exercised in the presence of the experiences being referred to or their imaginative recreations, phenomenal concepts use the experiences they refer to (by assumption, imaginative recreations of experiences are phenomenologically similar to actual experiences).

7 Loar $(1997,1999)$ argues in a similar way that we cannot expect to be able to see a priori that phenomenal and physical concepts corefer, even though both kinds of concepts refer directly, without the need for contingent reference-fixers. This expectation is, according to Loar, unjustified, because phenomenal and physical concepts play different cognitive roles (phenomenal concepts, unlike physical concepts, belong to the class of recognitional concepts, that is, type-demonstratives grounded in our dispositions to classify things by way of perceptual discriminations). I do not think that Loar's account of cognitive differences between phenomenal and physical concepts is correct, since I do not think that phenomenal concepts are demonstratives. Demonstratives have a reference-fixing "character" that leaves their referent open (Kaplan 1989). By contrast, our core phenomenal concepts are tied a priori to specific sorts of qualities (Chalmers 2003). Still, I agree with Loar's general idea that cognitive differences between phenomenal and physical concepts make the expectation of transparency in the case of phenomenal and physical concepts unjustified. The expectation at issue is unjustified when we think of phenomenal and physical concepts as directly referring concepts (which is what Loar argues) but also when we think of phenomenal and physical concepts as epistemically rigid concepts, as I argue here. 
epistemically rigid, even if we assume that we do not know a priori that phenomenal concepts refer to microphysical properties. Papineau's explanation turns on the point that phenomenal concepts use the experiences they refer to, in the sense that our exercises of phenomenal concepts are typically accompanied by the experiences being referred to or by their imaginative recreations (see note 6). Even though we do not know a priori that phenomenal concepts refer to microphysical properties, argues Papineau, phenomenal concepts are epistemically rigid because of the fact that they use their referents.

In reply, I agree that the use-mention feature of phenomenal concepts explains why phenomenal concepts are epistemically rigid. But I do not see why the use-mention feature of phenomenal concepts should explain why it is consistent to hold both that phenomenal concepts are epistemically rigid and that we do not know a priori that they refer to microphysical properties. After all, one might now ask why phenomenal concepts use their referents, even though we do not know a priori that they refer to microphysical properties. However, it is hard to see what the relevant explanation could be here (Papineau does not provide any such explanation). If so, Papineau's way of responding to the expectation of transparency does not look promising. We can explain why phenomenal concepts are epistemically rigid (in terms of the use-mention feature of phenomenal concepts), but we seem to have no explanation of why it is consistent to hold both that phenomenal concepts are epistemically rigid and that it is not true a priori that phenomenal concepts refer to microphysical properties.

Still, in order to show that the expectation of transparency in the case of phenomenal and microphysical concepts is unjustified, we do not need to explain why phenomenal concepts are epistemically rigid, even though we do not know a priori that they refer to microphysical properties. It is sufficient to appeal to the fact that the corresponding phenomenal and microphysical concepts play different cognitive roles. That is the strategy we have adopted here.

We have explained away one potential objection to the view that phenomenal and microphysical concepts are distinct epistemically rigid concepts of the same thing, namely, the objection based on the expectation of transparency. Is there any room for further objections? One might still 
worry that it is arbitrary to suppose that phenomenal and microphysical concepts are distinct epistemically rigid concepts of the same thing. As we have agreed, there are no uncontroversial examples of distinct epistemically rigid concepts of the same thing. If phenomenal and microphysical concepts are examples of such concepts, this example will be unique. But then one might think that postulating such an exception is entirely arbitrary and therefore unacceptable.

I do not think that the above worry is justified. The exceptional status of the phenomenal-physical case is not arbitrary at all. In fact, the reason why this is so is already implicit in what has been said so far. Ask yourself the following question: why cannot we make sense of there being conceptually distinct epistemically rigid concepts of the same thing outside the mindbody domain? Well, we cannot make sense of this because of the expectation of transparency. We expect that nonphenomenal epistemically rigid concepts cannot refer to the same thing unless they are connected a priori. This expectation of transparency in the case of nonphenomenal epistemically rigid concepts is perfectly intelligible. However, the corresponding expectation is not justified in the case of phenomenal and microphysical concepts, given that those concepts play different cognitive roles. So, this explains why it is not arbitrary to suppose that phenomenal and microphysical concepts are unique in being conceptually distinct epistemically rigid concepts of the same thing. The reason why we cannot make sense of uncontroversial examples of distinct epistemically rigid concepts of the same thing outside the mind-body domain simply does not extend to the particular case of phenomenal and microphysical concepts.

To illustrate this, think of the following case. The concept being a figure with all of its surface points equidistant from its centre is an epistemically rigid concept of sphericity: it refers to sphericity in all possible worlds considered as actual. Can we think of an epistemically rigid concept of sphericity that would be a priori distinct from the above concept? It seems that we cannot. We expect that any epistemically rigid concept of sphericity should be a priori connected with the concept a figure with all of its surface points equidistant from its centre. For example, consider the concept being disposed to roll when pushed. This is also an epistemically rigid concept of sphericity. But it is not difficult to see that there is an intelligible connection 
between being a figure with all of its surface points equidistant from its centre and being disposed to roll when pushed. In the absence of such a connection, we would have no reason to think that the two concepts at issue are concepts of the same thing. ${ }^{8}$ By contrast, we cannot expect that the connection between phenomenal and microphysical concepts should be transparent in the same sense. As we argued above, the radically different cognitive roles of those concepts will keep them unconnected a priori, even if those concepts refer - epistemically rigidly - to the same properties.

Now, one might argue that the case of phenomenal and microphysical concepts is not really different from the case of our two concepts of sphericity. Just as phenomenal and microphysical concepts play different cognitive roles, the concepts of sphericity also play different cognitive roles despite the fact that there is an intelligible connection between them: the former has its place in theoretical mathematical reasoning and the latter plays a role primarily in the practical concerns of everyday life. If this is right, then we haven't made the case that the expectation of transparency is unjustified in the case of phenomenal and microphysical concepts - the fact that phenomenal and microphysical concepts play different cognitive roles does not make this expectation unjustified. We should expect that there is an intelligible connection between phenomenal and microphysical concepts just as much as we expect that there is an intelligible connection between our concepts of sphericity. ${ }^{9}$

In reply, I do not think that the concepts of sphericity in question can be said to play different cognitive roles. I take it that two concepts of the same thing play different cognitive roles if the applications of those concepts put us in different kinds of cognitive relation to the referent. ${ }^{10}$ But the kind of cognitive relation we have to sphericity when we use the concept being a figure with all of its surface points equidistant from its centre is not

8 For a similar analysis of this example, see (Goff 2017, sec. 5.6).

$9 \quad$ I want to thank an anonymous reviewer for raising this point.

10 This can be illustrated by the concepts cramp and muscle contraction. Both are concepts of muscle contraction. But the former is a recognitional concept (discriminates muscle contraction perceptually), whereas the latter is a theoretical concept (describes muscle contraction in theoretical terms). (For an analysis of recognitional concepts, see Loar 1997).

Organon F 27 (3) 2020: 395-410 
different from the kind of cognitive relation we have to sphericity when we use the concept being disposed to roll when pushed. After all, both concepts refer to sphericity by way of describing what it takes for an object to be spherical. By contrast, there is a crucial cognitive difference between phenomenal and microphysical concepts: the former, unlike the latter, do not refer to experiences by way of describing them. Instead, they refer to experiences by way of using them (in the sense introduced by Papineau and mentioned in note 6 ). So, there is a clear sense in which the phenomenalphysical case is different from the case of concepts of sphericity. Given the lack of cognitive differences between our concepts of sphericity, we can expect that there should be an intelligible connection between those concepts. By contrast, we cannot expect that there should be an intelligible connection between phenomenal and microphysical concepts.

In the end, the case of phenomenal and microphysical concepts is special due to the unique status of phenomenal concepts among nonphenomenal epistemically rigid concepts in general. Nonphenomenal epistemically rigid concepts typically refer by way of describing their referents (think here of our concepts of sphericity), whereas phenomenal concepts do not refer by way of describing their referents. This explains why the expectation of transparency is unjustified uniquely in the case of phenomenal and microphysical concepts. When we think of nonphenomenal epistemically rigid concepts of the same thing, we expect that such concepts should be connected a priori, since we expect that there should be an a priori connection between epistemically rigid descriptions of the same thing. However, we cannot expect an a priori connection between phenomenal and microphysical concepts, given that phenomenal concepts, unlike physical-theoretical concepts, do not refer via descriptions.

I conclude that there is no good reason to think that phenomenal and microphysical concepts cannot corefer despite being distinct epistemically rigid concepts. If so, there is no good reason to think that the corresponding phenomenal and microphysical concepts cannot have coinciding primary intensions despite being distinct concepts. Consequently, there is no good reason to think that $P \& \sim Q$ cannot be 1-impossible despite being conceivable.

One could still raise the following objection to our reply to the twodimensional argument. We have argued that there is no good reason to 
think that the corresponding phenomenal and microphysical concepts cannot refer to the same properties despite being distinct epistemically rigid concepts. But one could argue that those concepts cannot corefer on the grounds that there cannot be the gap between the conceivability and 1possibility of $P \& \sim Q$. By assumption, if the corresponding phenomenal and microphysical concepts refer to the same properties despite being distinct epistemically rigid concepts, phenomenal and microphysical concepts have coinciding primary intensions despite being distinct concepts. This implies further that $P \& \sim Q$ is 1-impossible despite being conceivable. Thus, if there is a good reason to think that $P \& \sim Q$ cannot be 1-impossible despite being conceivable, this reason will count as a reason to think that the corresponding phenomenal and microphysical concepts cannot corefer despite being distinct epistemically rigid concepts after all.

No doubt, the above line of thought is well taken. It is, of course, true that if there is a good reason to think that $P \& \sim Q$ cannot be 1-impossible despite being conceivable, this will give us a good reason to think that phenomenal and microphysical concepts cannot corefer despite being distinct epistemically rigid concepts. But why think that $P \& \sim Q$ cannot be 1-impossible despite being conceivable? Whether $P \& \sim Q$ can be 1-impossible despite being conceivable comes down to whether the corresponding phenomenal and microphysical concepts can corefer despite being distinct epistemically rigid concepts. If this is so, then if we can show that there is no good reason to think that the corresponding phenomenal and microphysical concepts cannot corefer despite being distinct epistemically rigid concepts, we will have shown that there is no good reason to think that $P \& \sim Q$ cannot be 1-impossible despite being conceivable. But that is precisely what we have shown here. In effect the objection considered here does not work. Our response to the two-dimensional argument remains unthreatened.

I conclude again that premise 2 of the two-dimensional argument is unjustified. For this reason, the two-dimensional argument fails. Chalmers justifies premise 2 by the principle that ideal conceivability entails 1-possibility $(\mathrm{CP})$, but given that premise 2 is unjustified, $\mathrm{CP}$ is unjustified as well. Speaking more generally, then, the two-dimensional argument fails because the principle $\mathrm{CP}$ is unjustified. 


\section{Acknowledgements}

Thanks to an anonymous reviewer for very helpful comments.

\section{Funding}

I acknowledge the financial support of the National Science Centre, Poland/ project nr UMO-2017/25/B/HS1/02986.

\section{References}

Chalmers, David. 1996. The Conscious Mind: In Search of a Fundamental Theory. Oxford: Oxford University Press.

Chalmers, David. 2002a. "Consciousness and Its Place in Nature." In Philosophy of Mind: Classical and Contemporary Readings, edited by David Chalmers, 147272. Oxford: Oxford University Press.

Chalmers, David. 2002b. "Does Conceivability Entail Possibility?" In Conceivability and Possibility, edited by Tamar Gendler, and John Hawthorne, 145-200. Oxford: Oxford University Press.

Chalmers, David. 2003. "The Content and Epistemology of Phenomenal Belief." In Consciousness: New Philosophical Perspectives, edited by Quentin Smith, and Aleksandar Jokic, 220-72. Oxford: Oxford University Press.

Chalmers, David. 2006. "Two-Dimensional Semantics." In The Oxford Handbook of the Philosophy of Language, edited by Ernest Lepore, and Barry Smith, 574606. Oxford: Oxford University Press.

Chalmers, David. 2010. "The Two-Dimensional Argument against Materialism." In The Character of Consciousness, edited by David Chalmers, 141-92. Oxford: Oxford University Press.

Chalmers, David, and Frank Jackson. 2001. "Conceptual Analysis and Reductive Explanation." Philosophical Review 110 (3): 315-61. https://doi.org/10.1215/003 18108-110-3-315

Goff, Philip. 2017. Consciousness and Fundamental Reality. New York: Oxford University Press. https://doi.org/10.1093/oso/9780190677015.001.0001

Kallestrup, Jesper. 2006. "Physicalism, Conceivability, and Strong Necessities." Synthese 151 (2): 273-95. https://doi.org/10.1007/s11229-004-7325-9

Kaplan, David. 1989. "Demonstratives". In Themes from Kaplan, edited by Joseph Almog, John Perry, and Howard Wettstein, 481-563. New York: Oxford University Press.

Loar, Brian. 1997. "Phenomenal States". In The Nature of Consciousness: Philosophical Debates, edited by Ned Block, Owen Flanagan, and Güven Güzeldere, 597-616. Cambridge, MA: MIT Press. 
Loar, Brian. 1999. "On David Chalmers's The Conscious Mind." Philosophy and Phenomenological Research 59 (2): 465-72. https://doi.org/10.2307/2653684

Papineau, David. 2002. Thinking about Consciousness. Oxford: Oxford University Press.

Papineau, David. 2007. "Phenomenal and Perceptual Concepts." In Phenomenal Concepts and Phenomenal Knowledge: New Essays on Consciousness and Physicalism, edited by Torin Alter, and Sven Walter, 111-44. Oxford: Oxford University Press. https://doi.org/10.1093/acprof:oso/9780195171655.003.0007 